\title{
The second law of thermodynamics IN THE CONTEXT OF CONTEMPORARY PHYSICAL RESEARCH
}

Ivan A. Karpenko - PhD in Philosophy, Associate Professor.

National Research University Higher School of Economics. 20 Myasnitskaya St., Moscow 101000, Russian Federation; e-mail: gobzev@hse.ru

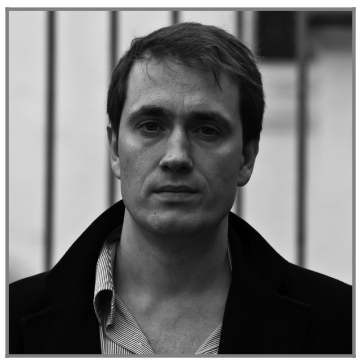

The second law results in the growth of the entropy - in superficial interpretation this principle presumes that the sufficient energy inevitably turns into the substandard energy. Order turns into chaos over time; however, chaos also turns into order under certain circumstances. The first research objective is to establish the possible prescientific ideas about the phenomenon - some philosophical intuitions that have preceded the scientific discovery of the second law and have conformed to it in a certain sense. It is essential because there are always certain bonds and continuity in the history of philosophy and science - the correct interpretation of the phenomenon becomes difficult, if not impossible, without the establishment of such bonds.

Moreover, the main task is to understand what the second law is and which significance its principal corollaries have. We need to give the second law a correct interpretation that will allow making assumptions about its connection with time in the context of the initial state problem and about the possible new ways of modern physics development - in particular, the creation of the quantum theory of gravity. Two solutions to the entropy and initial state connection problem are proposed in the context of the time arrow discussion ( $G$. Calender's approach to solving the problem is disputed).

Keywords: second law of thermodynamics, entropy, cosmology, arrow of time, gravity, quantum fluctuations

\section{ВТОРОЙ ЗАКОН ТЕРМОДИНАМИКИ В КОНТЕКСТЕ СОВРЕМЕННОЙ ФИЗИКИ}

\author{
Карпенко Иван \\ Александрович - кандидат \\ философских наук, доцент. \\ Национальный исследова- \\ тельский университет \\ «Высшая школа экономики». \\ Российская Федерация, \\ 101000 , г. Москва, \\ ул. Мясницкая, д. 20; \\ e-mail: gobzev@hse.ru
}

Второй закон термодинамики выражается в росте энтропии в поверхностной интерпретации этот принцип предполагает, что качественная энергия неизбежно превращается в некачественную энергию. Порядок со временем превращается в хаос, однако хаос также превращается в порядок - при особых обстоятельствах.

В статье устанавливаются некоторые донаучные представления о феномене - философские интуиции, которые предшествовали научному открытию второго закона и возможно идейно имели с ним корреляции, с тем чтобы корректно интерпретировать его следствия в контексте современной физики. Это существенно, потому что в истории философии и науки всегда существуют определенные связи и преемственность без установления таких связей правильное и актуальное истолкование явления становится затруднительным, если не невозможным. Основная цель исследования заключается в корректном понимании Второго закона и какое значение имеют его основные следствия. Для этого необходимо дать Второму 
закону правильную интерпретацию, которая позволит сделать предположения о его связи со временем в контексте проблемы начального состояния и о возможных новых путях развития современной физики, в частности квантовой гравитации. Предлагаются два решения проблемы связи энтропии и начального состояния в контексте дискуссии о стреле времени.

Ключевые слова: второй закон термодинамики, энтропия, космология, стрела времени, гравитация, квантовые флуктуации

\section{Problem Statement}

The second law of thermodynamics has been the subject of lots of studies. Here are some of the most relevant monographs, which deal with both the history of the issue and the contemporary significance of the law and its statements: [Attard, 2012; Blundell, Blundell, 2009; Borgnakke, Sonntag, 2012; Grandy, 2008; Greven, Keller, Warnecke (ed.), 2003]. However, these studies mostly research the problem from only physical positions regardless of any philosophical interpretation that would establish the meaning and significance of the phenomenon from a philosophical point of view. In other words, the second law and its consequences do not generally have disclosure in the historical and philosophical contexts.

We can define the entropy as the measure of orderliness (or disorderliness). Since it is evident that the concept of the disorder itself is vague and ideational, it could be defined as a minimum level of order (that is, in fact, the absence of order). However, in this case, we should specify the orderliness as a way to avoid the tautological definitions.

It is difficult to talk about entropy without connecting it to time because all the physical processes take place in time (the problematic of time and its possible connection with entropy are very important here, which will be discussed separately) ${ }^{1}$. Moreover, it is not clear whether time itself is a physical process ${ }^{2}$ since there is no clear and unambivalent definition of time. The connection in a strong sense means that it is one and the same or one is a corollary of the other. The probability of such a connection exists. The point is that the concept of "time" is purely ideational, reflecting the man's ideas about something which has been generating since ancient times - something inequable and transitory throughout life, about destruction and self-organization. Actually, it may have been an intuitive idea of entropy and evolution. This is a very bold

According to the problem of time in the context see the recent works [Callender, 2017; Bardon, Dyke (eds.) 2013; Albert, 2003; Whitrow, 2004; Arshinov, 2013, pp. 424; Sevalnikov, 2018, pp. 73-77; Karpenko, 2016; Landes, 2000; Smolin, Unger, 2014].

2 For example, the space-time is under review in the General relativity theory, but it can be hardly called the process. 
statement, and I do not intend to defend it, but my point is that the earlier intuitions, which in some generalized and primitive form designate some complex phenomena that later receive mathematical study and experimental description, may precede the scientific discoveries.

The ancient philosophers have already had an idea of something that we now call the fundamental laws of nature. These have been the notion of some principles, the arches that govern the world. Interestingly, in some cases, these principles have meant exactly what we would now call the self-organization or the growth of entropy - the laws, which establish the order ${ }^{3}$ and eventually pass it into the disorder.

Anaximander`s apeiron is that beginning - it origins everything and everything obeys the rule of "justice": at the appointed time objects turn into what they arise of.

Empedocles names the two fundamental principles - love and strife. While love prevails, the self-organization occurs - the connection of elements into the complex structures. But then strife begins to prevail, and everything breaks into primary components. The Empedocles's universe is cyclical: chaos replaces order and so on ad infinitum.

Philolaus shows another example: he mentions such a fundamental principle as harmony, which puts the substance into shape. Anaxagoras offers one more fundamental principle - Nous (Mind).

Even earlier mythological ideas, which have been later developed in philosophy, theology, and science, have contained the ideas about a certain arche, responsible for the management of ordered structures (and the ideas that claim the existence of a certain principle responsible for the destruction).

We could find many examples, but it is crucial that some primary, primitive intuitions about what we now call the low-entropic state and the growth of entropy have existed for a long time (of course, these are not the exact correlations).

Of course, if many different dictionaries contain different meanings of the terms, it becomes extremely difficult to prove that terms are the same. The problem of choosing a dictionary occurs - since terminology does not really reflect or describe phenomena, it turns out to be just a matter of agreement between scientists. For example, to describe physical reality one can use the terms "particle" or "field" (or string, ripples, etc.), can talk about the gravitational attraction or the curvature of space. All these are intuitively different things, but they apply to the same physical reality, which can be described in different terms but still lead to the same results of experiments.

Another example is the whole Democritus's intuition that the world is atoms and emptiness. His idea seems correct to us although it is formally entirely wrong considering the meanings of Democritus's atoms

3 In general, the fundamental law, which determines the origin of the order, does not exist. 
and emptiness. However, the modern Standard Model of Elementary Particle Physics [Nagashima, 2013; Schwartz, 2013] is a mathematical apparatus that allows us to effectively describe the observed reality while, in fact, it understands particles as points that can be infinitely small ${ }^{4}-$ these are not physical objects but the mathematical abstracts. The oneelectron theory of Feynman and Wheeler [Feynman, 1949, pp. 749-759] could be just as an exotic example. This theory states that there is only one electron traveling back and forth in time and allows us to make reasonable predictions about our reality.

Significantly, it is possible to describe the physical reality in various ways, which allow us to give the correct, consistent with the results of the experiments. This means that the physical reality itself is not yet clear to us, same with whether it can be established unambivalently ${ }^{5}$. The ancient philosophers have also intuitively understood this by mentioning that feelings do not give us reliable knowledge (Parmenides, Zeno, Melissus, skeptics, Plato and Aristotle have references to this, similar ideas can be traced in both Medieval and Modern times philosophies). Even the idea of George Berkeley that the existence means being perceptible does not mean that the very perceived reality is the true reality. It can be made up and consists of what we are just able to perceive [Berkeley, 1978, p. 326]. Kant articulates the problem most clearly by distinguishing the phenomenal and the noumenal - the knowable and the uncognazable, things in themselves. In this context, his idea that time and space are a priori forms of sensibility, but not the real physical objects is very essential [Kant, 1994, pp. 56-58].

Therefore, we need to be extremely careful while bonding the entropy, which is a quite clearly defined concept in physics, and the time, which is something that precedes any strict scientific concepts, vague and intuitive. Moreover, we stumble upon the mentioned above inevitable problem while interpreting the phenomenon of entropy from a philosophical

4 This is the reason of failure to bond the quantum field theory and the General relativity theory and to create a gravity theory of full value. The Standard Model assumes that particles could be arbitrarily small, so the quantum fluctuations increase indefinitely, and the GR space drops its smoothness while particles' reduction to ever smaller scales. All that leads to the infinities in the solutions of the combined equations of both theories. The superstring theory tries to solve this problem by imposing restrictions on the particles sizes that means turning them not into the abstract mathematical objects but the actual physical ones with their limiting length (Planckian length).

5 The question is whether the reality should somehow be unambivalent. Different (mathematical) descriptions may be simultaneously true. This means that our intuitive model of the fixed physical reality, which must have the only correct terms to be somehow unambivalently described in, may turn out to be itself incorrect (the duality of completely different theories, discovered by Edward Witten, Juan Martín Maldacena and others, for example, proves the above mentioned, see more on this further). 
point of view - if it is, in fact, possible for us to understand what is behind this phenomenon and how our intuitions approach the essence of the matter ${ }^{6}$.

\section{The Core of the Second Law}

It is well known that in 1824 the engineer Sadi Carnot has formalized the second law of thermodynamics while handling the tasks of a purely practical nature, without any relation to basic science. He has found that the steam engine working process is irreversible - a certain amount of energy is always lost during its operation. Later, Rudolf Clausius has defined the concept of entropy. His idea has been based on the statement, which is a truism for us - the heat can never pass from a colder to a warmer body. A reverse process occurs - the equalization of temperatures in time, which is irreversible. That is, a colder body will not become even colder, but only hotter - during their interaction.

Clausius has realized that he had discovered the new law of nature. It can be formalized as follows: the entropy of an isolated system either remains constant or increases in time. Entropy is a specific quantity: the amount of heat divided by body temperature. Entropy reaches a maximum when the temperature equalizes (equilibrium).

Before Ludwig Boltzmann, no one had associated entropy with atoms. It had been considered as some kind of separated independent force. Atomism has not been popular. Boltzmann has proposed a new formulation, which is consistent with modern ideas. His idea identifies the entropy as the number of microscopic system states, which are indistinguishable from a macroscopic point of view. Such an understanding is possible only through the acceptance of atomism. Let's have a case imaginary experiment in order to clarify it. Following the practice of Eurytus as of Aristotle's records [Aristotle, 2016, 1092b, pp. 14-15] we take a set of small stones. For example, four of them. We will throw them and each time they will fall differently and sometimes (rarely) form the correct square. All other states are chaotic for us and therefore indistinguishable from each other, but the only one is in order. Now we take a set of a hundred stones in order to get the shape of a horse. While pouring them again and again to the ground we will never get the shape of a horse (or will, but we would have to wait for a long time, perhaps longer than the Universe exists). All these piles with variously located stones are indistinguishable to the observer. But when we finally get a horse shape, we will

6 In favor of science, it is important to mention that the approaching is nevertheless close - the very successful in experiments theories are the evidence of that. We discuss more towards if this approximation is infinite. 
distinguish it from the pile. Similarly, a sand pile can be poured over and over again and all these piles will be the same, they will not fold into something other than a pile - for example, into a sandcastle. This is the core of Boltzmann entropy: there are many more ways to create disorder than to create order.

Entropy here may seem like just a focus reflecting a lack of knowledge about the entire system. If we could keep track of all the stones and the sand piles then, of course, all the piles would be different for us. This is indeed the case. But the matter is that Boltzmann has introduced the elementary particles into the case, which are just impossible to keep track of.

The Heisenberg's uncertainty principle is the matter. It states that simultaneous measurement of the particle momentum and its position are impossible. The more accurately we define one of the parameters, the more uncertain the other becomes. The wave function describes the particle's behavior, the Schrödinger equation - its evolution over time. It is basically impossible for us to know the location and the speed of movement (including direction) of the particle before the measurement. After the measurement the particle's previous way and location are indefinite, we cannot turn the system into the initial state (on the problem of measurement in the quantum mechanics see [Belavkin, 2000, pp. 101129; Braginsky, Khalili, Thorne (ed.), 1992; Greenstein, Zajonc, 2005; Wheeler, Zurek (eds.), 2014], i.e., this process is irreversible ${ }^{7}$.

The only probabilistic ${ }^{8}$ prediction is available - we can predict the particular result in most cases. In other words, the microcosm is unobservable in an uncontrollable state. Our measurement violates its free state and gives us nothing (from the classical-physical point of view) about its initial state. The quantum fluctuations (the constant birth and annihilation of virtual particles) affect the state of the microcosm and complicate everything with these basically random processes.

Thus, the complete knowledge of the microscopic systems is impossible. That is, the microscopic states are basically indistinguishable macroscopically. The more elements are in the system, the more sets of indistinguishable states are there.

7 In connection to this, it is suggested that the quantum measurement determines the arrow of time (since it is irreversible). However, classical physics (Newton's mechanics) is completely reversible. Its laws work in both directions, it does not distinguish the direction of time.

8 It is important from a mathematical point of view that this is not about the classical probability, but about the complex-valued. 


\section{Entropy and Time}

Henri Poincaré in his famous recurrence theorem ([Poincaré, 1890, pp. 1270], proof by [Carathéodory, 1919, pp. 580-584], version for quantum mechanics [Schulman, 1978, pp. 2379-2380]) has shown that any system will return to its initial state after a particular time. The recurrence time of the above mentioned four elements (a square of stones) system is relatively small - there are not many ways to arrange them in a closed loop. However, the recurrence time also increases with the number of elements. In the system of a hundred elements it is enormous - up to the billions of years. Nevertheless, it is important that any system, even a very complex one, will return to its initial state according to the theorem - you just need to wait long enough (maybe infinitely from a mathematical point of view). But this means the following: high entropy state will return to a low entropy state in some time. A contradiction surely known to Poincare is obvious here - according to the second law of thermodynamics the entropy of an isolated system either increases or remains unchanged. But his theorem has stated that a washed away by the sea sandcastle would reappear sooner or later, i.e., a high-entropic state will inevitably turn into a low-entropic one.

The acceptance of the cyclic model of the universe ${ }^{9}$ can resolve this contradiction. According to the idea of the theorem, which is, by the way, consonant with the idea of the Nietzsche's eternal recurrence, the system should return to the same state, but it will also turn into all its various possible configurations including inevitable reiterations on the way to it. This makes perfect sense: with regard to the infinite time, any finite set of elements must reconfigure to all possible variations including infinite reiterations and the fluctuations turn to be the exiting the high-entropic state - the random low-entropic inevitable in the statistical mechanics' configurations.

Here lies the problem of a bond between entropy and time. The fact is that the transition from a high-entropic state to a low-entropic state does not mean a time reversal. While observing the self-organization of the matter, we do not observe (and basically could not observe) the passage of the reversed time, the turnaround of the arrow of time.

The following case imaginary experiment will help us to indicate the possible bond between entropy and time. We need to try to imagine that time has stopped. A separate question is whether it is conceivable at all (if, for example, we adopt the Kantian position that time is an a priori

9 The idea of the cyclical universe is a very early one and has been already mentioned by the ancient philosophers (Pythagoras, Epicurus, the Stoics, and others), as well as in various mythologies. 
form of sensuality then the stopped time means the perception termination, the death of the observer).

Nevertheless, let us assume that time has stopped ${ }^{10}$. Is it possible to imagine that some processes continue to occur then? There is only a frozen moment ${ }^{11}$ of the present, a static blink, the future does not come, it does not become the past. Of course, it is impossible to observe this moment because the observation is a process and all processes that we know from a macroscopic point of view occur in time. But let's suppose that it has happened. In this case, no processes occur. But the absence of processes is comparable with the maximum entropy state that is when the temperature is close to absolute zero. At this temperature no processes can occur, the entropy is maximum. However, the universe with the maximum possible entropy does not actually seem to have an absolute zero temperature due to quantum fluctuations - it will be very low, but higher than zero. By the way, this perhaps explains the impossibility of the "frozen moment" - a state of absolute zero cannot be reached. It seems to be possible to associate entropy with time, but there is one problem. Quantum fluctuations are the processes and all processes, as we have assumed, occur in time. But since they are always happening, then time cannot basically stop.

Aristotle, not following Plato, has defined time through the movement (the time is a measure of movement [Aristotle, 2018, IV, p. 11]). There is a certain sense here in the context of our mental experiment. But in this case, as follows from our conclusions, time is itself a kind of process that cannot be stopped. Then the question arises: could this process ever have a beginning if it cannot have an end?

Augustine is worth recalling here with his solution of the problem of God`s activities before the creation of the universe [Augustine, 2009, 11, $\mathrm{X}, \mathrm{p} .12$ ] - according to him time has appeared along with the creation of the universe, so there is no point in asking what had been before since there had been no "before". But what does absence of time mean? According to our logic - the absence of entropy.

If we admit an inflationary model of the origin of the universe the processes, which are the cause of inflation, have taken place in the inflaton's field (false vacuum) before the Big Bang, probably caused the time/entropy. However, can these processes (if they can even be called the processes) themselves occur in time? If so then the false vacuum has a non-zero (and low) entropy. The universe originates as a result

10 In theory, this is real - moving in space at the speed of light is just enough. The only problem is that the mass of an object does not allow doing this.

11 The lack of the clear explanation of "the frozen moment of the present" becomes another problem. It appears that only acceptance of the time discreteness makes it possible to explain. The problem implicitly arises in Zeno's paradoxes, Augustine has mentioned it, etc. (see an interesting review [Koyré, 1985, pp. 27-51]). 
of symmetry violation when various interactions (at first, gravitational, then others: strong, weak and electromagnetic) fall out of the initial common interaction. I.e., this initial state - before the origin of the universe is entirely symmetrical and, therefore, relatively low-entropic because of the connection between the orderliness and the entropy.

However, the problem is that entropy must increase (according to the second law of thermodynamics) both into the past and into the future. Therefore, it should increase from the present moment in both directions and here the bond with time loses meaning. It turns out that if the arrow of time is characterized as an entropy growth, then both directions - forward and backward - are our future. But the truth is that we remember the past and do not remember the future. Our consciousness maintains such a characteristic that memory imprints the states with low entropy, but we do not know the future - we cannot "see" the states with the predictable higher entropy (the disorder will increase).

Appealing one more time to Kant, it could basically be reckoned as just a characteristic of our consciousness - to arrange the events in a certain way in time and space, but this does not concern the real events. This idea is also consonant with the General relativity theory where the notion of a single space-time is under the broad review. Here is the violation of the principle of simultaneity - the past and the future are not universal, they depend on the observer's point of view - on his or her position, speed and direction of movement, local curvatures of space-time. In the GR universe all the time points and events are already initially preset in a sole unit of space-time and what happened for one observer in the past is happening for another one now, for the third one it will still be happening (on the other side, they cannot inform each other). In this sense, consciousness can only perceive the events, which are already preset in spacetime, locating them on its internal scale of the past/future according to some reason.

But if we accept the point of view that the past is objective, our memory is real, then the entropy in the past should have been low. Otherwise, our memories are not real but the result of fluctuation (very rare but inevitable in the unlimited time $)^{12}$. In other words, if entropy increases from the present moment into the past and the future then our memories of how the sandcastle has been washed away, the glass has been broken,

12 It would be rather willing to assume that there is a certain threshold value for the fluctuations quantity and the large-scale fluctuations cannot exist. The periodicity of fluctuations, which are locally lowering the entropy, could be detected by graphing an equation of fluctuations for a container with gas. In general, the entropy remains high, and there is no chance to observe the large-scale macroscopically distinguishable fluctuations (for example, such as all gas gathering at the one wall). However, this assumption is not possible to justify yet. 
the egg has spread 13 are not real because, in fact, we must remember the reverse processes. So, I, we, including all our memories or the whole universe, are the result of the rare fluctuation (the spontaneous connection of elementary particles into the ordered structures), which has arisen all at once (perhaps, right now). It is difficult to accept such a hypothesis - it is hardly probable.

It is important to highlight some alternative points of view on the time problem before we go further to the proposed solution of the problem. These are the ones presented in two articles by Craig Callender. The first one is devoted to the time arrow [Callender, 1997, pp. 223234]. The second one covers the "problem" of the initial low entropy state and develops the ideas of the first one (the "problem" is in quotes here because Callender considers this more likely a false problem than a real one) [Callender, 2004, pp. 240-255]. His first article justifies that the problem of time direction is the result of an error in the foundations of statistical mechanics - more specifically, in its initial hypotheses and formulations. The second article relies on the works of Huw Price [Price, 1996], where he uses a similar approach and concludes that the initial state problem is a consequence of the initial axioms and their following explanations. I. e., the hypothesis of an improbable initial state is our incorrect interpretation of physics and thus it needs improvement. Here Callender offers the three solutions: "(a) re-write the dynamics so that the Past State would be generic in the solution space of the new dynamics, (b) add a new non-dynamical law of nature, or (c) eliminate the measure making such states abnormal. In other words, we could make the Past State likely (as a result of new dynamics or not) or make it 'a-likely'”. Thus, he proposes to redo the foundations of the theory in such a way that the initial state does not become improbable but "normal". I disagree with him at this point because the initial interpretation of the theory seems to me quite correct and the numerous experiments prove this. Nevertheless, the possible Callender's verity still needs to be considered. As an analogy, we have various interpretations of quantum mechanics: no matter which one we choose for the basis, the experimental results stay the same. This suggests a complex philosophical discourse, which is the subject for separate studies. I assume here that the basic hypotheses are true ${ }^{14}$.

13 This particularly shows that entropy was lower in the past - we never see the sand castle being built by itself, the whole glass gathers out of its fragments, etc.

14 In a recent work according the same problem [Goldstein, Tumulka, Zanghi, 2016] the authors follow an alternative path, justifying that the low entropy state associates with the time arrow and can be same justified, rather than postulated. But, unlike Callender, they use the justification not of an epistemological nature for this but of a mathematical one ("Toy Model"). 
The idea of Mathias Frisch [Frisch, 2010, pp. 13-33] is also worth noting: the cause-effect asymmetry caused by the arrow of time is not related to the physical conditions that determine the second law of thermodynamics and these are, in fact, different processes. Thus, the entropy growth and the time run are divided ${ }^{15}$.

\section{Two Ways to Solve the Problem of the Initial State}

The hypothesis about the low-entropic state of the Universe at the moment of its origin seems more plausible. This theory explains the arrow of time, the characteristic of our memory and the observations results. But the problem is that this hypothesis itself needs validation - there are no obvious reasons why the entropy was low in the distant past since it must increase indefinitely both into the past and into the future.

I offer two solutions. The first one is a hypothesis about the nature of time. It is possible to consider time as a kind of fundamental entity, which has been primarily preset before the origin of the universe. From this point of view, it is extremely difficult to explain the low entropy in the past because, as mentioned before, in this case, the entropy should increase both into the past and into the future. But it is possible (and quite reasonable, considering that the space-time structure in the initial singular state is unknown to us and it is only clear that it was not the same as in the observed state) to assume that time in the usual sense arises together with the Universe. As already mentioned before, Augustine has been the first to make such an assumption (from an obvious theological point of view God has created the world and the time). Thus, if we connect the arrow of time and the entropy, then it is obvious that the latter should have been low in the past because the selected reference point had appeared the beginning of time. This is justified because the selected point of reference is macroscopically distinguishable. It certainly differs from all other possible subsequent states and is therefore low-entropic (towards to these other states). It is reasonable to assume directly that the end of time is a state with the highest possible entropy - the universe with a temperature close to absolute zero. Eventually, the system can be assumed to return to its original state with low entropy (for example, as a result of fluctuations), which will mean a newly selected time reference point. However, again, time will not go backward with a decrease in entropy (the local entropy decrease can explain this - for example, we still do the work by

15 Patrick Stokes [Stokes, 2010, pp. 485-507] has a very interesting study on this subject from a purely philosophical point of view: the author tries to show that the problem of asymmetric perception of time is a key problem of the Kierkegaard's "or/or". 
adding up the stones into a horse shape figure and building a sandcastle and the total entropy increases).

If we reject the discussion whether the time is some fundamental, eternal value or arises at some point and is a property of matter, another way is to analyze the initial state of the universe, namely the Big Bang.

We have used to define entropy as a measure of disorder. However, this is actually not entirely correct. Let's replace the stones from our example with gas molecules. If they are collected in a small area of space (for example, in the middle of a container) then, of course, the entropy of this state will be lower than if they spread in a free state all over the container. The most interesting is that the more we tighten the region of space, i.e., put these molecules into the smaller space, the less will be the entropy of this state (since we thereby reduce the number of possible relocations in space). Let's now suppose that the molecules have formed the figure of a horse. This is certainly a macroscopically distinguishable low-entropic state. But the fact is that there are far more ways to arrange molecules into a horse figure than to collect them in a very small area of space (less than a horse figure). Therefore, the entropy of the second system is smaller. However, we will specifically call the first system (horse) the ordered one. Here lies the direction for a possible solution to the problem of a low-entropic initial state of the universe.

The solution is probably in the Big Bang ${ }^{16}$. The Big Bang involves several phase transitions, during which radiation and matter form. At a certain stage, the gravity also arises (more precisely, it separates from other interactions). The gravity begins to play an independent role in the interval between $10^{-43}$ and $10^{-42}$ seconds from the beginning of the universe (even before the inflationary expansion) when symmetry is broken. We are particularly interested in this moment (the end of the Planckian era) since the common beliefs of space-time may not make sense at smaller sizes.

The bond between entropy and order has been discussed above. The early state of the universe - the state of a hot plasma lump - is difficult to call the ordered one in the usual sense and it is tempting to say that this is a state with very high entropy. However, the gravitational effects do play a role here (including, of course, the level of quantum gravity). The gravity makes the structures form macroscopically distinguishable configurations, so the matter behaves differently in the gravity presence than in its absence. It loses its homogeneity and begins to gather into clumps as affected by the gravity (the elements attract each other, the clusters of elements attract more strongly). Galaxies, stellar systems, stars and planets are formed from gas-dust clouds as a result of the gravitational

16 We are talking about the hypothesis of the universe origin in the context of the inflationary model proposed by A. Gut [Guth, 1997]. 
interaction ${ }^{17}$. Thus, the presence of gravity is capable of explaining the low-entropic initial state.

While discussing the connection between gravity and entropy Callender [Callender, 2010, pp. 34-58] also follows a different path with assumption that there is a well-defined Boltzmann entropy, which is able to increase in some self-gravitating systems. However, he makes a proviso concerning this being more of a philosophical hypothesis liable for thorough research. Wallace [Wallace, 2010, pp. 513-540] answers him rightly noting among other things that the role of gravity in the calculation of entropy should be distinguished from the entropy of gravity itself. This is an important note that we would also like to point out.

Concerning such a solution to the problem, it should be mentioned that gravity determines the arrow of time, and here we have to raise the question of the bond between gravity, entropy and time. Such a statement of the question involves the quantum gravity - the gravitational effects in the microworld (the quantum description of gravity). The area has been little researched so far, but some promising ideas do exist, which will be discussed in conclusion.

\section{Conclusion}

The bond between entropy and gravity (and gravity is directly related to time, the GR is an example) is very promising from the point of view of building a theory of quantum gravity ${ }^{18}$. Obviously, the effects of quantum gravity should play a significant role at the early stages of the origin of the universe - when it has had the parameters of Planckian values. The black holes are the objects, for a description of which the quantum gravity is of particular importance.

The black hole is the limit of gravitational compression of matter. In other words, the given area of space can imbed a black hole of a given size and accordingly a certain amount of entropy (the entropy of black holes is maximum ${ }^{19}$ ). This means that the gravity imposes a limit on the amount of entropy that can be contained in a particular region of space.

Based on the work of Jacob Bekenstein, Stephen Hawking has established [Hawking, 1974, pp. 30-31] that the entropy of a black hole equals to a quarter of its horizon area (in Planckian units, a Planckian length unit

\footnotetext{
17 It is important that as a result of the appearance of these structures, which seem to be increasingly ordered (compared with the initial state of a hot plasma with gravity), the entropy increases as it should.

18 See, for example, concerning the problem of constructing a theory of quantum gravity [Herbert, 2009; Oriti (ed.), 2009; Vincent (ed.), 2012].

19 In general, the entropy of black holes radiation is higher than their entropy and the entropy of entirely empty space is even higher.
} 
can be considered as one bit of information). Thus, the entropy (and information) is connected not with the volume of a black hole but with its surface. Later this has allowed Leonard Susskind and Gerard 't Hooft to show that information about the object is not contained in the object (in the sense of its volume) but on its surface [Susskind, 1995, pp. 63776396], ['t Hooft, 1993, web]. For example, all information about a threedimensional object is contained on its two-dimensional surface (this has got the name of the holographic principle).

Based on this result ${ }^{20}$, Juan Maldacena has established the duality of two theories of different dimensions, one of which contains the gravity and the other does not [Maldacena, 1999, pp. 1113-1133]. He has shown that in accordance with the holographic principle the language of a theory of another dimension (in a simpler mathematical apparatus), where there is no gravity, can describe the gravitational theory. This is a purely theoretical result that describes the models that are not literally applicable to our world. But its importance is that due to the equivalence of theories it allows us to describe the quantum gravity indirectly ${ }^{21}$.

The quantum effects are non-local - they act instantly (the bond between a remote surface and its projection of a higher dimension is nonlocal). From my point, the nonlocality of the quantum gravity is probably a significant argument in favor of the low entropy in the initial state when these effects have played a key role. The classical ideas about space-time bonds are not effective when nonlocality appears (in particular, the entanglement of particles expresses it) - all the events turn out to be interdependent. The reason for non-locality lies perhaps in the multidimensionality of space at the micro level, emphasized in the theory of superstrings (see on the multidimensionality in theory [Zwiebach, 2009; Yau, Nadis, 2010]), where the additional dimensions open up the new space-time bonds.

As I have shown, on larger scales the gravitational effects create the conditions for the low entropy - heterogeneity 22 , providing a low entropy start and an arrow of time. The low entropy start basically may be preceded by a state with high entropy or may not - the state before "creation" is entirely symmetrical ${ }^{23}$, and it is not at all clear whether such a state should be considered low-entropic or not. Returning to Poincare's theorem, my conclusion is that the state with maximum entropy (when all

20 And also on the important result of Edward Witten [Witten, 1995, pp. 85-126].

21 See the important reflection on the bond between the quantum entanglement and the gravity [Karpenko, 2018].

22 The studies of the relic radiation show that these heterogeneities may be a consequence of the early quantum fluctuations, but this does not negate the role of the gravitational effects.

23 Meaning that the fundamental interactions have not been separated and the recently observed symmetry violations have not yet happened. 
the black holes have evaporated and only an empty homogeneous space of elementary particles remains) goes to the state with low entropy, which becomes a new reference point - because of quantum fluctuations and gravitational effects. The credibility of this scenario becomes higher if we consider that we are not talking about some incredibly low-entropic state (there is no need for it). The entropy of the initial state is still high, but lower than it becomes after ${ }^{24}$.

\section{References / Список литературы}

Albert, 2003 - Albert, Z. Time and Chance. Cambridge: Harvard University Press, 2003, $192 \mathrm{pp}$.

Aristotle, 2016 - Aristotle. Metaphysics. Indianapolis: Hackett Publishing Company, 2016, $712 \mathrm{pp}$.

Aristotle, 2018 - Aristotle. Physics. Indianapolis: Hackett Publishing Company, 2018, 420 pp.

Arshinov, 2013 - Arshinov, V.I. "Vremja-kommunikacija-Vselennaja" [TimeCommunication-Universe], in: V. Kazytinski (ed.). Metavselennaja, prostranstvo, vremja [Metaverse, Space, Time]. Moscow: Institut filosofii RAN, 2013, pp. 4-24. (In Russian)

Attard, 2012 - Attard, P. Non-Equilibrium Thermodynamics and Statistical Mechanics: Foundations and Applications. Oxford: Oxford University Press, 2012, $480 \mathrm{pp}$.

Augustine, 2009 - Augustine. Confessions. Oxford: Oxford University Press, 2009, 352 pp.

Bardon, Dyke (eds.), 2013 - Bardon, A., Dyke, H. (eds.) A Companion to the Philosophy of Time. Wiley-Blackwell, 2013, 600 pp.

Belavkin, 2000 - Belavkin, V. "Dynamical Solution to the Quantum Measurement Problem, Causality, and Paradoxes of the Quantum Century”, Open Systems and Information Dynamics, 2000, vol. 7 (2), pp. 101-129. sian)

Berkeley, J. Sochinenija [Compositions]. Moscow: Mysl, 1978, 556 pp. (In Rus-

Blundell, Blundell, 2009 - Blundell, S.J., Blundell, K.M. Concepts in Thermal Physics. Oxford: Oxford University Press, 2009, 516 pp.

Borgnakke, Sonntag, 2012 - Borgnakke, C., Sonntag, R. Fundamentals of Thermodynamics. New York: Wiley, 2012, 912 pp.

Braginsky, Khalili, Thorne, 1992 - Braginsky, V., Khalili, F.Y., Thorne, K.S. (ed.) Quantum Measurement. Cambridge: Cambridge University Press, 1992, 212 pp.

Callender, 1997 - Callender, G. "What Is 'The Problem of the Direction of Time’?”, Philosophy of Science, 1997, vol. 64, no. 4, pp. 223-234.

24 For example, the initial state could be $10^{88}$, now it is $10^{101}$ and the maximum is $10^{120}$. Obviously, there is a huge difference between these values, but $10^{88}$ is not a low entropy. 
Callender, 2004 - Callender, G. “There Is No Puzzle About the Low-Entropy Past", in: Hitchcock, C. (ed.). Contemporary Debates in Philosophy of Science. Bodmin: Blackwell, 2004, pp. 240-257.

Callender, 2010 - Callender, G. “The Past Hypothesis Meets Gravity”, in: Hüttemann, A. \& Ernst, G. (eds.). Time, Chance and Reduction. Cambridge: Cambridge University Press, 2010, pp. 34-58.

Callender, 2017 - Callender, G. What Makes Time Special. Oxford: Oxford University Press, 2017, 336 pp.

Carathéodory, 1919 - Carathéodory, C. “Über den Wiederkehrsatz von Poincaré”, Sitzungsberichte der Preussischen Akademie der Wissenschaften, 1919, pp. 580-584.

Feynman, 1949 - Feynman, R. "The Theory of Positrons", Physical Review, 1949, vol. 76 (6), pp. 749-759.

Frisch, 2010 - Frisch, M. "Does a Low-Entropy Constraint Prevent Us from Influencing the Past?”, in: Hüttemann, A. \& Ernst, G. (eds.). Time, Chance and Reduction. Cambridge: Cambridge University Press, 2010, pp. 13-33.

Goldstein, Tumulka, Zanghi, 2016 - Goldstein, S., Tumulka, R., Zanghi, N. Is the Hypothesis About a Low Entropy Initial State of the Universe Necessary for Explaining the Arrow of Time? [https://arxiv.org/pdf/1602.05601.pdf, accessed on 19.02.2020].

Grandy, 2008 - Grandy, W.T. Entropy and the Time Evolution of Macroscopic Systems. Oxford: Oxford University Press, 2008, 256 pp.

Greenstein, Zajonc, 2005 - Greenstein, G.S., Zajonc A.G. The Quantum Challenge: Modern Research On The Foundations Of Quantum Mechanics. Burlington: Jones \& Bartlett Learning, 2005, 296 pp.

Greven, Keller, Warnecke, 2003 - Greven, A., Keller, G., Warnecke, G. Entropy. Princeton: Princeton University Press, 2003, 384 pp.

Guth, 1997 - Guth, A. The Inflationary Universe: The Quest For A New Theory Of Cosmic Origins. New York: Basic Books, 1997, 358 pp.

Hawking, 1974 - Hawking, S. "Black Hole Explosions”, Nature, 1974, 248 (5443), pp. 30-31.

Herbert, 2009 - Herbert, H.W. Quantum Gravitation: The Feynman Path Integral Approach. New York: Springer Publishing, 2009, 342 pp.

Kant, 1994 - Kant, I. Kritika chistogo razuma [Kritik der reinen Vernunft]. Moscow: Mysl, 1994, 591 pp. (In Russian)

Karpenko, 2015 - Karpenko, A. "V poiskah real'nosti: Ischeznovenie” [In Search of Reality: Disappearance], Filosofiya nauki - Philosophy of Science, 2015, no. 20, pp. 36-72. (In Russian)

Karpenko, 2016 - Karpenko, I.A. "Problema interpretatsii ponyatiya vremeni v nekotorykh kontseptsiyakh sovremennoi fiziki" [Concept of Time Problem Interpretation in Several Modern Physics Theories], Voprosy filosofii, 2016, no. 9, pp. 72-83. (In Russian)

Karpenko, 2018 - Karpenko, I.A. "Filosofskaya interpretatsiya sovremennykh podkhodov k sozdaniyu kvantovoi teorii gravitatsii" [Philosophical Interpretation of Modern Approaches to the Creation of Quantum Theory of Gravity], Filosofija nauki i tehniki - Philosophy of Science and Technology, 2018, vol. 23, no. 1, pp. 54-67. (In Russian) 
Koyré, 1985 - Koyré, A. “Zametki o paradoksah Zenona” [Notes on Zeno’s Paradoxes], in: Koyré, A. Ocherki istorii filosofskoi mysli [Essays on the History of Philosophical Thought]. Moscow: Progress, 1985, pp. 25-71. (In Russian).

Landes, 2000 - Landes, D. Revolution in Time: Clocks and the Making of the Modern World. Cambridge: Harvard University Press, 2000, 502 pp.

Maldacena, 1999 - Maldacena, J. “The Large N Limit of Superconformal Field Theories and Supergravity”, International Journal of Theoretical Physics, 1999, vol. 38, no. 4, pp. 1113-1133.

Nagashima, 2013 - Nagashima, Y. Elementary Particle Physics: Foundations of the Standard Model, vol. 2. New York: Wiley, 2013, 646 pp.

Oriti, 2009 - Oriti, D. (ed.) Approaches to Quantum Gravity. Toward a New Understanding of Space, Time and Matter. Cambridge: Cambridge University Press, 2009, 604 pp.

Poincaré, 1890 - Poincaré, H. "Sur le problème des trois corps et les équations de la dynamique. Divergence des séries de M. Lindstedt”, Acta Mathematica, 1890, vol. 13 (1-2), pp. 1-270.

Porus, 2018 - Porus, V.N. "Kontekstualizm v filosofii nauki" [Contextualism in the Philosophy of Science], Epistemology \& Philosophy of Science, 2018, vol. 55, no. 2, pp. 75-93. (In Russian)

Price, 1996 - Price, H. Time's Arrow and Archimedes' Point: New Directions for the Physics of Time. Oxford: Oxford University Press, 1996, $322 \mathrm{pp}$.

Schulman, 1978 - Schulman, L.S. "Note on the Quantum Recurrence Theorem", Physical Review A, 1978, vol. 18 (5), pp. 2379-2380.

Schwartz, 2013 - Schwartz, D. Quantum Field Theory and the Standard Model. Cambridge: Cambridge University Press, 2013, 859 pp.

Sevalnikov, 2018 - Sevalnikov A. "Vremya v kvantovoy teorii" [Time in Quantum Theory], Metafizika - Metaphysics, 2018, no. 1 (27), pp. 73-77. (In Russian)

Smolin, 2013 - Smolin, L. Time Reborn: From the Crisis in Physics to the Future of the Universe. Boston: Houghton Mifflin Harcourt, 2013, 352 pp.

Smolin, Unger, 2014 - Smolin, L., Unger, R.M. The Singular Universe and the Reality of Time. Cambridge: Cambridge University Press, 2014, 565 pp.

Stokes, 2010 - Stokes, P. "Fearful Asymmetry: Kierkegaard's Search for the Direction of Time”, Continental Philosophy Review, 2010, vol. 43, no. 4, pp. 485-507.

Susskind, 1995 - Susskind, L. "The World as a Hologram”, Journal of Mathematical Physics, 1995, vol. 36, no. 11, pp. 6377-6396.

't Hooft, 1993 - 't Hooft, G. Dimensional Reduction in Quantum Gravity. [https://arxiv.org/pdf/ gr-qc/9310026v2.pdf, accessed on 22.12.2019].

Vincent, 2012 - Vincent, F.R. (ed.) Classical and Quantum Gravity: Theory, Analysis and Applications (Physics Research and Technology). New York: Nova Science Pub Inc., 2012, 656 pp.

Wallace, 2010 - Wallace, D. "Gravity, Entropy, and Cosmology: in Search of Clarity”, British Journal for the Philosophy of Science, 2010, vol. 61, no. 3, pp. 513540.

Wheeler, Zurek, 2014 - Wheeler, J.A., Zurek, W.H. (eds.) Quantum Theory and Measurement. Princeton: Princeton University Press, 2014, 842 pp.

Whitrow, 2004 - Whitrow, G.J. What is time? The Classic Account of the Nature of Time. Oxford Oxford: University Press, 2004, 192 pp. 
Whitrow, 2004 - Whitrow, G.J. Time in History: Views of Time from Prehistory to the Present Day. Oxford, Oxford University Press, 2004, 229 pp.

Witten, 1995 - Witten, E. “String Theory Dynamics in Various Dimensions”, $\mathrm{Nu}$ clear Physics B, 1995, vol. 443, no. 1, pp. 85-126.

Yau, Nadis, 2010 - Yau, S.-T., Nadis, S. The Shape of Inner Space: String Theory and the Geometry of the Universe's Hidden Dimensions. New York: Basic Books, 2010, $400 \mathrm{pp}$.

Zwiebach, 2009 - Zwiebach, B. A First Course in String Theory. Cambridge: Cambridge University Press, 2009, 694 pp. 